\title{
Growth rates of dynamic dermal model exposed to laminar flow and magnetic fields
}

\author{
Luis Javier Martinez*, Carlos Rafael Pinedo, Jose Oscar Gutierrez, Hector Cadavid
}

\begin{abstract}
Introduction: Ongoing research in the use of electromagnetic stimulation as coadjuvant in fracture healing has led the authors to begin generating computer models in order to predict cellular growth changes when cells are electromagnetically stimulated. By generating these models, scientists will be able to better understand how electromagnetic fields affect cellular development. The experimental design integrated a cellular culture bioreactor along with an external magnetic stimulation system, which allowed for dermal models to be exposed to controlled magnetic fields. Methods: Initially, it was necessary to analyze the static growth of Normal Human Skin Fibroblast (NHSF) cells when they were exposed to Extremely Low Frequency - Electromagnetic Fields (ELF-EMFs). Using optimal conditions for the NHSF culture, from stimulation signal to scaffolding material, we were able to perform the dynamic flow stimulation experiments. Results: The following systems were developed: (1) a bioreactor aimed at cellular tissue culture, and (2) Helmholtz coils capable of generating stimulation signals for the cultured tissue. The authors were able to appreciate the quantified values of cellular density diluted in all the experiment samples that were taken and overall, the irradiated samples displayed an average increase of 53\% higher cellular density for the same amount of initial cellular seeding when the cells were exposed to a $1 \mathrm{mT}, 60 \mathrm{~Hz}$ magnetic field signal. Conclusion: ELF-EMF's indeed alter NHSF cell growth rates and it is the challenge of the authors to continue investigating what cellular mechanisms are altered when cells are exposed to ELF-EMF's.
\end{abstract}

Keywords: Bioelectromagnetics, Magnetic fields, Bioreactor, Helmholtz coils, Biomedical applications of radiation.

\section{Introduction}

Long bone fractures such as the femur are very common in Colombia, mainly due to car-motorcycle and motorcycle-motorcycle traffic accidents. Many of these fractures do not heal in the expected window of time after treatment, which raises socio-economic concerns for both the patients and society (MartinezRondanelli et al., 2014). Doctors have had to explore different ways to promote or decrease recovery time for fracture union. One of the methods explored has been surrounded by controversy in medical literature, which is the use of electromagnetic fields to promote bone healing. Electromagnetic stimulation consists of using a device that generates an electromagnetic field as a non-invasive way to improve fraction union success. Clinical trials have explored the effects of electromagnetic stimulation in fracture healing with contradictory results. Some clinical trials report that fraction union times are decreased when fractures are exposed to electromagnetic fields (Cook et al., 2015; Iryanov and Kiryanov, 2015; Li et al., 2007; Massari et al., 2015; Moncada et al., 2011), while other trials report that fraction union times actually increase when the fractures were exposed to the electromagnetic fields with respect to the control studies (Handoll and Brorson, 2015; Shi et al., 2013). Whether fraction union times decrease or increase it seems that one thing is certain and that is that there is some sort of effect occurring to the normal reproduction of cells in the body when they are exposed to the electromagnetic fields. Recent studies have explored the possibility of electromagnetic stimulation promoting different cellular processes including synthesis of growth factors, collagen fibers, proteoglycans, and cytokines.

In order to better understand how electromagnetic fields interact with human body cells, a sterile environment where human body cells could grow and be exposed to electromagnetic fields to be studied was proposed for construction. This sterile environment would be called a bioreactor and it would be a space where different cellular structures could be exposed and then tested to better understand the cellular mechanisms that are being activated or promoted when exposed to the electromagnetic fields. The main difference between cellular culture in a petri dish and in a bioreactor is that inside a bioreactor the cellular tissue would allow for three-dimensional growth, which gravity restricts 
when growing cellular tissue in a petri dish. Therefore by constructing a bioreactor and being able to grow cellular tissue in three dimensions, the possibility of eventually growing cellular tissue is a possibility.

The use of bioreactors, allows users to maintain the specific environmental conditions necessary for the proper survival of the tissue that is being cultivated (Griffith and Naughton, 2002; Martin et al., 2004; Martin and Vermette, 2005). Due to the fact that tissues, unlike cellular monolayers, need to grow in multiple layers, they begin to form a thickness and three-dimensional structure. Normally the most common technique used in research laboratories is to perform cellular experiments in petri dishes or culture plates, which allow investigators to assess individual cellular behavior. In the case of tissue culture, however, these petri dishes are not a viable option because they do not allow the cellular nutrients to disseminate throughout the thickness of the tissue. The in vitro growth of three-dimensional tissues requires the development of devices that can satisfy the physical and chemical requirements of the cellular mass growing inside the matrix (Griffith and Naughton, 2002). Amongst the main aspects in the development and implementation of a bioreactor for this specific purpose are cell nutrition in the matrix, control over the environmental conditions, long-term cellular culture maintenance, evaluation and control of the biological parameters and automation of the procedure (Vunjak-Novakovic, 2003).

The aim of using bioreactors to facilitate tissue engineering is to be able to recreate the most controlled environment possible in low gravity conditions in order to reproduce optimal growth conditions for cellular cultures in a three-dimensional environment (Alvarez Barreto, 2009), similar to the growth that occurs naturally in biological organisms. By being able to have such strict control of the multiple variables that affect tissue growth, we are then able to simplify the study of physiological changes in cells when a specific variable or set of variables are altered, in order to determine how it affects the normal cellular development.

Based on this rigorous control that we have over tissue growth cultured inside a bioreactor, we decided to expose the cells growing inside the bioreactor to electromagnetic fields in order to better understand the effects occurring to the normal reproduction of cells in the body when they are exposed to these fields. The bioreactor serves the purpose of allowing growth around a cellular matrix, as shown by Griffith and Naughton (2002) and Malda et al. (2003), which will be irradiated by a magnetic field from the outer part of the bioreactor. The main aspects of the development and use of the bioreactor are to provide nutrition of the cells, to gain control over the environmental conditions and long term maintenance of the cultures, and to control the evaluation of biological parameters. Various models have been developed in order to use extremely low frequency electromagnetic field (ELF-EMF) stimulation to induce cellular growth and alter proliferation growth rates, by increasing the cellular expression of specific growth factors in different cellular culture models, such as those by Focke et al. (2010) and Martino et al. (2010a, 2010b).

We had previously studied the possibility of combining these two lines of research and develop a cellular culture bioreactor coupled with external magnetic stimulation coils in order to study the effects of magnetic field exposure on tissue growth of a skin model, this study was published by Restrepo et al. (2013) where the computational model of the integration of these two systems is presented. The main objective is to achieve proper function of the bioreactor for the culture of cellular tissues coupled with an external electromagnetic stimulation system, with the endpoint of recreating an ideal environment, which would allow for the culture and growth of cells in a sterile and favorable environment for the development of tissue grafts exposed to controlled electromagnetic fields. The present study aims at implementing and materializing said study and initiating tests with cellular models.

\section{Methods}

Previous studies carried out by our group explored the static growth rates of the proposed skin model exposed to ELF-EMFs when using traditional cellular culture techniques. Also, the skin model was validated by analyzing whether appropriate cellular growth occurred on the Chitosan-covered polylactic acid (PLA) scaffolds used for the tissue growth and cellular viability tests were carried out. After these preliminary experiments were successful, we were able to proceed with our end goal of creating an integrated bioreactor with an external electromagnetic stimulation system as a favorable environment for the development of skin grafts exposed to controlled electromagnetic fields.

\section{Cellular culture}

The required cellular lines were extracted and then maintained in the laboratory environment required for the cells to have adequate growth conditions. Cell line CRL-1474 [CCD-25Sk], which is a cell line of normal human skin fibroblasts (NHSF), was used. The cell line was used to explore whether different intensities of ELF-MFs can exert altered 
proliferation effects other than the classical apoptosis. The cell lines were acquired and remain available in the IN-VITRO Cell Culture Laboratory cell bank in the School of Pharmacology in the Health Faculty at the Universidad del Valle.

The procedure to cultivate NHSF cells on bioorganic-synthetic scaffolds began by seeding the cells at a concentration of $7 \times 106$ cells on the scaffold support on a petri dish and allowing it 2 hours to adhere along with a D-MEM culture medium supplemented with $10 \%$ calf FBS, $100 \mathrm{U} / \mathrm{mL}$ of penicillin, $100 \mu / \mathrm{mL}$ of streptomycin, $2 \mu \mathrm{g} / \mathrm{mL}$ of amphotericin, which were incubated with a $5 \% \mathrm{CO} 2$ humid atmosphere at $37^{\circ} \mathrm{C}$. Once the primary fibroblast cultures reached confluence, they were inactivated using Mitomycin C at a concentration of $4 \mu \mathrm{g} / \mathrm{mL}$ for 2 hours. The isolated cells were then cultured inside the bioreactor with the scaffold supports at a concentration of $2 \times 106$ over a monolayer of inactivated fibroblast cells $(6 \times 106)$ and cultured in a humid atmosphere with $5 \% \mathrm{CO} 2$ at $37^{\circ} \mathrm{C}$. The cell culture growth medium is made up of D-MEM and Ham-F12 at a 3:1 proportion supplemented with 10\% FBS (Hyclone), insulin $(5 \mu \mathrm{g} / \mathrm{mL})$, hydrocortisone $(0.4 \mu \mathrm{g} / \mathrm{mL})$, triiodothyronine $(1.3 \mathrm{ng} / \mathrm{mL})$, choleric toxin $(8 \mathrm{ng} / \mathrm{mL})$ (Sigma), adenine $(24 \mu \mathrm{g} / \mathrm{mL})$, penicillin $(100 \mathrm{U} / \mathrm{mL})$, and streptomycin $(100 \mu \mathrm{g} / \mathrm{mL})$. The culture medium was replenished every 2 to 3 days and after the first medium change, some epidermal growth factor (EGF, $10 \mathrm{ng} / \mathrm{mL}$ ) was also added to the medium. This was the protocol that was mirrored when attempting to treat the cultured growth tissue (Ferreira et al., 2010; Price et al., 2001; Reid et al., 2007).

\section{Electromagnetic stimulation}

When we refer to the electromagnetic stimulation system, we are taking into account the set-up of two pieces of equipment that, in conjunction, make up the magnetic stimulation system. The first equipment basically consists of the source system where the magnitude, frequency and waveform are defined for the generated magnetic field, while the second equipment is the irradiating system where the geometry of the final element is defined for the magnetic field as well as for the positioning of the biological system within the area of interest for the generated field. Both the signal generating and control equipment and the magnetic field generator are described in the work developed by Restrepo et al. (2013).

The magnetic stimulation system includes a variable voltage source which allows the user to infer the frequency and the intensity of the stimulation signal and includes a pair of Helmholtz coils as the final element of magnetic irradiation. This system satisfies the electrical as well as the physical characteristics necessary to support the current, voltage and magnetic field specifications for the extremely low frequency range. In addition, the system source input has regulation characteristics, power dispersal and the necessary protection in order to guarantee the produced signal. The system does not have a backup during the power phase in the case of electrical starvation. Figure 1 shows the first prototype of the magnetic stimulation system.

\section{Bioreactor}

The bioreactor has a Couette-Taylor design which is made up of two concentric cylinders: the external cylinder consists of a constant rotation powered by an external motor while the internal cylinder is maintained static and the cellular growth medium flows in and out and provides the necessary nutrients for adequate growth of the tissue, as well as proper removal of cellular respiration products. This system satisfies the electrical and physical characteristics required to support the operating conditions needed by the system (continuous, sterile functioning). Figure 2 is an image of the bioreactor system prototype. The bioreactor system prototype was developed by the research group in high voltage (GRALTA) of the Universidad del Valle as part of a research project funded by an internal call for projects by Pinedo et al. (2011).

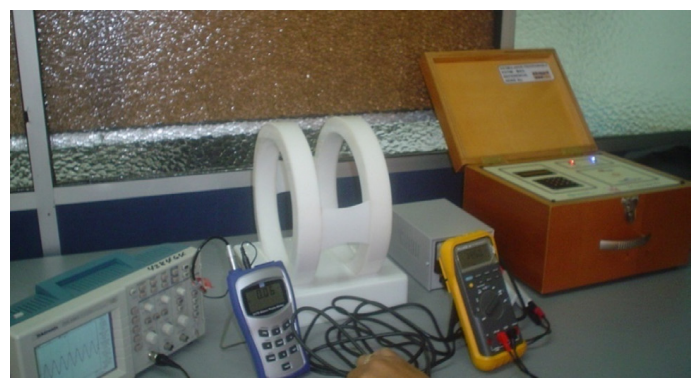

Figure 1. Image of Helmholtz coils and power generating system.

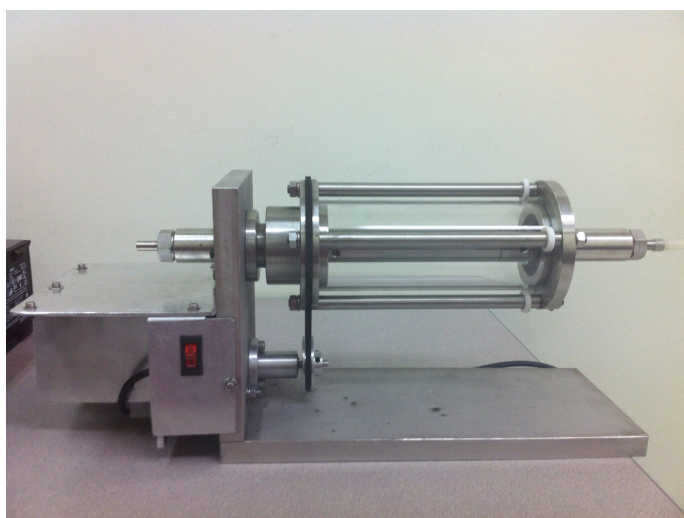

Figure 2. The constructed bioreactor with coupled rotating motor. 


\section{Integration of the systems}

For the actual systems, certain devices have to be considered, such as auxiliary equipment like the signal generator for the coils, which is part of the electromagnetic stimulation system, and auxiliary equipment for the bioreactor, such as the motor, $\mathrm{O} 2$ and $\mathrm{CO} 2$ tanks, flow pumps (tubes and valves), etc. For the integration of the two systems, the placement of the bioreactor at the center of the Helmholtz coils is explored, as seen in Figure 3. Now the experiments for tissue culture were carried out in order to begin correlating results, with the future possibility of clinical studies.

However, once the tissue is being cultured and grown in the bioreactor, we must keep in mind the research performed by Litovitz et al. (1994), in which a series of experiments were developed from which it was established that at least two mechanisms surround the effects of time-varying low intensity magnetic fields on animal cells. The first is through Faraday's law of induced electric fields, which consequently induces an electrical current within the cells experiencing the field, and which give the same results as those obtained when the current is applied directly through electrodes with the same current density. The second mechanism indicates that the direct current (DC) magnetic field generated from the application of an alternating current (AC) signal also influences the observed effects. Thus, if we are starting from the principle that there exists an induced current traveling through the exposed cells, and due to the complex nature of how cells are asymmetrically arranged within themselves, one must assume that a variable that has to be considered is the cellular tissue growth in relation to the orientation of the magnetic field. Different studies have concluded that exposure to magnetic fields can have different effects on growth rates depending on the orientation of

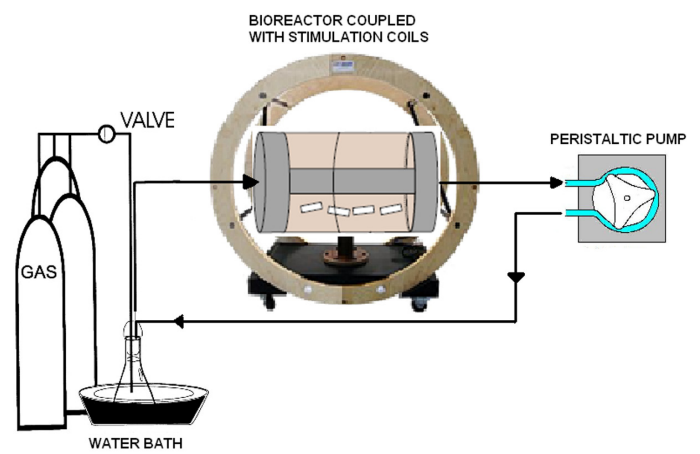

Figure 3. Schematic set-up of the different components making up the experimental system, including medium reservoir, stimulation coils, bioreactor, peristaltic pump, and gas containers. certain macromolecules in the cells, such as collagen. Human skin fibroblasts have also been shown to orient themselves based on collagen orientation with respect to the magnetic fields, (Miyakoshi, 2005).

The human skin fibroblast tissue, which was grown inside the bioreactor, based on its orientation, grew along the length of the bioreactor and the thickness of the tissue grew perpendicular to the axis of the cylindrical bioreactor. This orientation favors growth because the magnetic field is oriented in the same direction along the axis of the cylinder. It can be established that the field is oriented in such a direction because, since the Helmholtz coils are parallel in one dimension, this means that the resultant field will be perpendicular to the direction of the current. Therefore, the cylinder, the tissue and the magnetic field are all aligned. Since collagen aligns itself with the field, this property is ideal with respect to stimulation of growth, which is a desired property for the project's aims.

\section{Control samples}

When our group carried out the static growth stimulation experiments, it was necessary to also include a control sample with each experiment which was not exposed to any controlled magnetic fields. This was done because we needed baseline measurements in order to compare if there was indeed any change in growth when the cellular tissue was exposed to said magnetic fields. With each iteration of the experiment, a control sample was seeded from the same batch of cells in order to minimize confounding variables. Then for our dynamic flow growth stimulation experiments it was necessary to also culture and grow cellular tissue without controlled magnetic field exposure, so that we again had a baseline measurement against which to compare our stimulated tissue.

Initially, the equipment was setup and the system ran continuously for one week. For the non-stimulated tissue, the Helmholtz coils were placed but not energized, thus generating no magnetic field. When the week had elapsed, the scaffold was removed, and the cellular tissue was removed in order to measure the density of the cellular tissue that was present. Temperature was controlled by keeping the DMEM in a water bath at $37^{\circ} \mathrm{C}$, thus ensuring that in the 2 minutes it takes the medium to flow through the system it will not substantially lose temperature. The project aims that the changes in growth are due to the stimulation and not temperature or shear stresses, because both the control and the stimulated tissue were subject to the same conditions, and repeated $n=4$ times; thus, all other variables being equal, changes should stem from the stimulation alone. 
The tissue was removed using $0.25 \%$ Trypsin, then neutralized, and the remaining cellular structure was diluted in $10 \mathrm{~mL}$ of DMEM. Also, $10 \mathrm{~mL}$ samples of the DMEM flowing within the system were taken from the medium reservoir in the water bath, the flow tubes and from inside the bioreactor itself, in order to count the cells and compare their density with that of samples growing on the scaffold. This was to allow us to determine if the tissue was growing on the scaffold, or if any sample was just that of the cellular tissue growing in the enclosed system.

\section{Experimental samples}

Once the results for the non-irradiated cellular tissue culture were obtained, we continued by performing the controlled magnetic field stimulation experiments for tissue under dynamic flow conditions. After obtaining the cellular tissue and preparing the bioorganic-synthetic scaffold, we setup the stimulation equipment and the bioreactor and seeded the cellular tissue and began the experiments. This time, however, the Helmholtz coils were turned on for a period of 4 hours each day during the course of the week-long time period, just like all the other stimulation experiments performed up until this point. Also, in the same manner as the previous non-stimulated experiments, at the end of the week, we removed the scaffold and detached the NHSF cellular tissue from the scaffold and diluted it in $10 \mathrm{~mL}$ of DMEM to measure cellular density. We also took samples from the reservoir, the tubes and the bioreactor cylinder itself, in order to compare cellular densities and ensure an actual difference in concentration being grown on the scaffold.

\section{Results}

Once the optimal culture conditions were established, we proceeded to the culture of the NHSF cells within the Couette-Taylor bioreactor coupled with the magnetic field stimulation system, in order to evaluate tissue growth within the established parameters. These results allowed us to identify the optimal characteristics for cellular culture conditions as well as adequate bioorganic-synthetic scaffolding for the possible development of skin grafts. Figure 4 shows the experimental set-up that was outlined in the previous Section II-D in Figure 3, as it was organized in the laboratory.

Figure 5 shows the quantified values of cellular density diluted in all the experiment samples that were taken, including the non-stimulated cellular tissue; in each case, the irradiated samples displayed an average increase of $53 \%$ higher cellular density for the same amount of initial cellular seeding. Actually,

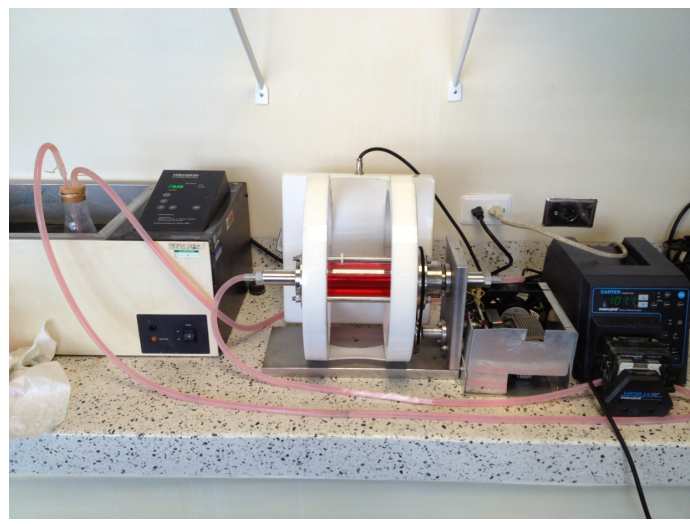

Figure 4. Couette-Taylor bioreactor set-up working with cellular tissue seeded on scaffold.

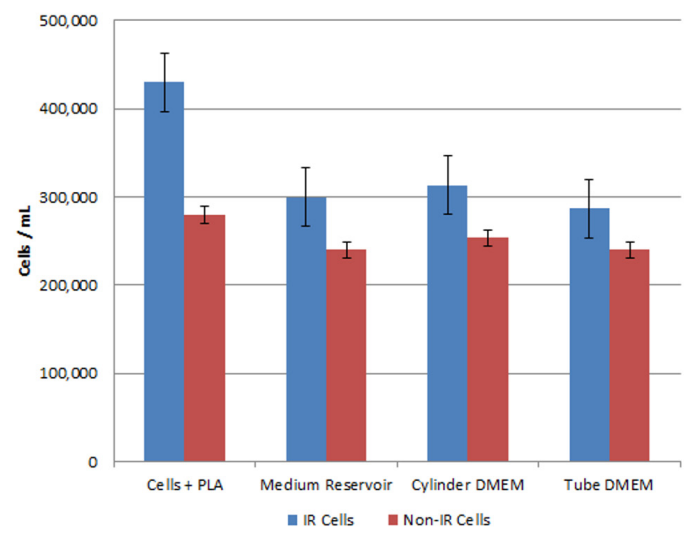

Figure 5. NHSF cellular density for irradiated and non-irradiated iterations.

the non-irradiated experiments were conducted with an initial seeding of 20,000 cells $/ \mathrm{mL}$, with average results of 280,000 cells $/ \mathrm{mL}$, whereas the irradiated experiments were conducted with an average initial seeding of 15,333 cells $/ \mathrm{mL}$, with average results of 430,000 cells $/ \mathrm{mL}$.

We can also observe from the cellular tissue density results that, even though there were higher densities in all the irradiated samples, not just on the scaffold, but also in the DMEM samples from other areas of the system, the growth rates were not as high as with the scaffold samples, thus showing that indeed only the target stimulated areas are displaying the increased growth rates, just like the intended consequences from performing the irradiations. The increase in the other areas of the system can be attributed to the fact that not just the sample is being irradiated, but also cells floating in the interstitial DMEM that are not attached to the material, but are nonetheless reproducing and being exposed to the stimulation. However, since they are not receiving the same amount of magnetic field waves, due to the constant DMEM interchange 
that is taking place due to the system flow, they are not growing at the same rate as the cells attached to the biomaterial scaffold.

\section{Discussion}

Based on the previous approximations modeled in the work by Restrepo et al. (2013), we were able to develop and build the a bioreactor design for a Couette-Taylor bioreactor with sufficient processing capacity to allow us to culture cells into biological tissue and allow for the required amounts of growth medium culture within the vessel required for a dermal model, while at the same time having a magnetic stimulation system coupled to its design.

The aforementioned computer model consisted of a three-dimensional coil system which helped the authors evaluate the generated magnetic field. The model verified the magnetic field distribution between the separated coils and their surroundings at different operating conditions, thus verifying the bioreactor and coils system design, and also succeeded in creating a computational model on which to estimate the electrical variables induced under different experimental conditions in vitro (Restrepo et al., 2013). Overall, we obtained a very accurate approximation of the behavior of the magnetic field and how it was acting upon our cellular tissue samples.

After having the validated computational model of the prototype and the magnetic fields, everything here on after belongs to this new project, which consists in the development of the physical prototype. The prototype bioreactor and coil system were developed and constructed for the University, either here at the University, as in the case of the coil system, or through third-party contractors with sufficient experience working with related designs, as in the case of the bioreactor. Even though the design was sufficient to perform the required experimental tests on cellular culture, we still observed and identified key new requirements relating to the conditions which should be met by future prototypes.

Once the equipment was setup and ready for work to be performed, and the cellular lines were sufficiently confluent, the experimental protocols were carried out and the experimental tests performed with the bioreactor under blank control and stimulation conditions. Following the experiments, growth rates were assessed and compared to determine differences in proliferation. Afterwards, the respective cell characterization tests were performed on the tissue in order to test for cell viability and compatibility and to compare them with the growth rates to look for patterns. The obtained results were analyzed and validated by comparing them with the experimental tests and procedures that were previously outlined. The results were characterized based on the stimulation variables that were applied on the cells in order to form a relationship between the cells and the simulation results. The results also helped us validate the experimental protocol and to improve the methodology for measuring the electrical properties of the stimulated cellular tissue. Despite both methodologies still not being completely accurate, they offer us the prospect of a constantly evolving procedure which opens the way to continue improving research techniques for in vitro tissue measurement that can lead to results based on more accurate and verifiable protocols and methodologies.

We compared the $53 \%$ growth rate change between irradiated cellular tissue and non-irradiated cellular tissue under flow rate conditions, to the growth rate changes for cellular tissue under static conditions which, before reaching $100 \%$ cellular confluence, exhibited growth changes from $20 \%$ to $38 \%$, thus having comparable changes between flow and static conditions. The reason we concluded that they are comparable rate changes, even though $53 \%$ is 15 points higher than the $38 \%$ change in static conditions, is that they are still in the same order of magnitude between $0-100 \%$ change, as opposed to values that would increase cellular growth by $10 \times$, or even some documented cases where changes can even range in the $100 \times$ variance. This allowed us to conclude that these are comparable results, which in turn let us continue performing our analysis of why we were observing these changes.

As initially stated in the introduction, the goal of this work was to establish a baseline for the actual changes that occur in different cellular cultures when exposed to ELF-EMF's. This work showed measurement techniques for electrical properties in the in vitro tissue, the correlations between growth rates and cellular mechanisms, the equipment measurements of magnetic fields and the elaboration of coils that can shield and generate frequencies at ambient and geomagnetic magnitudes, and the computational models developed to approximate intensity values of the MFs.

However this is only the beginning for this area of research. We previously stated that the ultimate end-point would be to find the underlying effect that is altering the normal reproduction of cells in the body when they are exposed to the electromagnetic fields. It is clear that cellular mechanisms are being altered based on studies that have explored the possibility of electromagnetic stimulation promoting different cellular processes including synthesis of growth factors, collagen fibers, proteoglycans, and 
cytokines. Future work should focus on tracking these different molecules inside cells in real time, in order to observe how electromagnetic fields alter their normal concentration levels and thus being able to correlate their variations to the variations in cellular growth rates.

\section{Acknowledgements}

This project was funded by the Colombian "Departamento Administrativo de Ciencia, Tecnología e Innovación-Colciencias" with a fellowship awarded for Ph.D. programs and was part of a internal call for research projects by the Investigations Vice-rectory of the Universidad del Valle 2011.

\section{References}

Alvarez Barreto JF. Regeneración ósea a través de la ingeniería de tejidos: una introducción. Revista de Estudios Transdisciplinarios. 2009; 1(2):98-109.

Cook JJ, Summers NJ, Cook EA. Healing in the new millennium: bone stimulators: an overview of where we've been and where we may be heading. Clinics in Podiatric Medicine and Surgery. 2015; 32(1):45-59. http://dx.doi. org/10.1016/j.cpm.2014.09.003. PMid:25440417.

Ferreira LM, Sobral CS, Blanes L, Ipolito MZ, Horibe EK. Proliferation of fibroblasts cultured on a hemi-cellulose dressing. Journal of Plastic, Reconstructive \& Aesthetic Surgery; JPRAS. 2010; 63(5):865-9. http://dx.doi.org/10.1016/j. bjps.2009.01.086. PMid:19345169.

Focke F, Schuermann D, Kuster N, Schär P. DNA fragmentation in human fibroblasts under extremely low frequency electromagnetic field exposure. Mutation Research. Fundamental and Molecular Mechanisms of Mutagenesis. 2010; 683(1-2):74-83. http://dx.doi.org/10.1016/j. mrfmmm.2009.10.012. PMid:19896957.

Griffith LG, Naughton G. Tissue engineering: current challenges and expanding opportunities. Science. 2002; 295(5557):1009-14. http://dx.doi.org/10.1126/science.1069210. PMid:11834815.

Handoll $\mathrm{HH}$, Brorson S. Interventions for treating proximal humeral fractures in adults. Cochrane Database of Systematic Reviews. 2015; 11:CD000434. http://dx.doi. org/10.1002/14651858.CD000434.pub4. PMid:26560014.

Iryanov YM, Kiryanov NA. Reparative osteogenesis and angiogenesis in low intensity electromagnetic radiation of ultra-high frequency. Vestnik Rossiiskoi Akademii Meditsinskikh Nauk. 2015; 70(3):334-40. http://dx.doi. org/10.15690/vramn.v70i3.1330. PMid:26495722.

Li F, Xu KW, Wang HC, Guo WY, Han Y, Liu B, Zhang RQ. Effects of static magnetic field on human umbilical vessel endothelial cell. Journal of Medical Colleges of PLA. 2007; 22(2):106-10. http://dx.doi.org/10.1016/S10001948(07)60023-9.

Litovitz TA, Montrose CJ, Doinov P, Brown KM, Barber M. Superimposing spatially coherent electromagnetic noise inhibits field-induced abnormalities in developing chick embryos. Bioelectromagnetics. 1994; 15(2):105-13. http://dx.doi.org/10.1002/bem.2250150203. PMid:8024603.

Malda J, Martens DE, Tramper J, van Blitterswijk CA, Riesle J. Cartilage tissue engineering: controversy in the effect of oxygen. Critical Reviews in Biotechnology. 2003; 23(3):175-94. http://dx.doi.org/10.1080/bty.23.3.175. PMid:14743989.

Martin I, Wendt D, Heberer M. The role of bioreactors in tissue engineering. Trends in Biotechnology. 2004; 22(2):80-6. http://dx.doi.org/10.1016/j.tibtech.2003.12.001. PMid:14757042.

Martin Y, Vermette P. Bioreactors for tissue mass culture: design, characterization, and recent advances. Biomaterials. 2005; 26(35):7481-503. http://dx.doi.org/10.1016/j. biomaterials.2005.05.057. PMid:16023202.

Martinez-Rondanelli A, Martinez JP, Moncada ME, Manzi E, Pinedo CR, Cadavid H. Electromagnetic stimulation as coadjuvant in the healing of diaphyseal femoral fractures: a randomized controlled trial. Colombia Médica (Cali, Colombia). 2014; 45(2):67-71. PMid:25100891.

Martino CF, Perea H, Hopfner U, Ferguson VL, Wintermantel E. Effects of weak static magnetic fields on endothelial cells. Bioelectromagnetics. 2010a; 31(4):296-301. PMid:20119972.

Martino CF, Portelli L, McCabe K, Hernandez M, Barnes F. Reduction of the earth's magnetic field inhibits growth rates of model cancer cell lines. Bioelectromagnetics. 2010b; 31(8):649-55. http://dx.doi.org/10.1002/bem.20606. PMid:20830734.

Massari L, Osti R, Lorusso V, Setti S, Caruso G. Biophysical stimulation and the periprosthetic bone: is there a rationale in the use of Pulsed Electromagnetic Fields after a hip or knee implant? Journal of Biological Regulators and Homeostatic Agents. 2015; 29(4):1013-5. PMid:26753669.

Miyakoshi J. Effects of static magnetic fields at the cellular level. Progress in Biophysics and Molecular Biology. 2005; 87(2-3):213-23. http://dx.doi.org/10.1016/j. pbiomolbio.2004.08.008. PMid:15556660.

Moncada ME, Sarmiento C, Martinez C, Martinez A. Magnetic stimulation for fracture consolidation: clinical study. Conference Proceedings of IEEE Engingeering in Medicine and Biology Society. 2011; 33(1):1141-4.

Pinedo CR, Cadavid H, Gutiérrez JO, Restrepo AF, Martinez LJ, Criollo W. Desarrollo de un bioreactor con estimulación magnética y validación en un modelo de implante de piel artificial. Proyecto de convocatoria interna. Cali: Universidad del Valle; 2011.

Price RD, Das-Gupta V, Frame JD, Navsaria HA. A study to evaluate primary dressings for the application of cultured keratinocytes. British Journal of Plastic Surgery. 2001; 54(8):687-96. http://dx.doi.org/10.1054/bjps.2001.3712. PMid:11728112.

Reid MJ, Currie LJ, James SE, Sharpe JR. Effect of artificial dermal substitute, cultured keratinocytes and split thickness skin graft on wound contraction. Wound Repair and Regeneration. 2007; 15(6):889-96. http://dx.doi. org/10.1111/j.1524-475X.2007.00313.x. PMid:18028138. 
Restrepo AF, Martinez LJ, Pinedo CR, Franco E, Cadavid H. Design study for a cellular culture bioreactor coupled with a magnetic stimulation system. IEEE Latin America Transactions. 2013; 11(1):130-6. http://dx.doi.org/10.1109/ TLA.2013.6502791.

Shi HF, Xiong J, Chen YX, Wang JF, Qiu XS, Wang YH, Qiu Y. Early application of pulsed electromagnetic field in the treatment of postoperative delayed union of long-bone fractures: a prospective randomized controlled study. BMC Musculoskeletal Disorders. 2013; 14(1):35. http://dx.doi.org/10.1186/1471-247414-35. PMid:23331333.

Vunjak-Novakovic G. The fundamentals of tissue engineering: scaffolds and bioreactors. Novartis Foundation Symposium. 2003; 249:34-46. http://dx.doi.org/10.1002/0470867973. ch4. PMid:12708648.

\footnotetext{
Authors

Luis Javier Martinez ${ }^{1,2^{*}}$, Carlos Rafael Pinedo ${ }^{3}$, Jose Oscar Gutierrez ${ }^{4}$, Hector Cadavid ${ }^{1}$

${ }^{1}$ High Voltage Research Group, School of Electrical and Electronic Engineering, Faculty of Engineering, Universidad del Valle, Cali, Colombia.

${ }^{2}$ Research Group GINIC-HUS, School of Biomedical Engineering, Faculty of Engineering, Universidad ECCI, Calle 51 \# 19-20, Bogota, Colombia.

${ }^{3}$ Perception and Intelligent Systems Research Group, School of Electrical and Electronic Engineering, Faculty of Engineering, Universidad del Valle, Cali, Colombia.

${ }^{4}$ Pharmacology Research Group, School of Basic Sciences, Medical Faculty, Universidad del Valle, Cali, Colombia.
} 